\section{CURSOS SUPERIORES DE TURISMO E HOTELARIA NO BRASIL}

\author{
Marilia Gomes dos Reis Ansarah
}

Mirian Rejowski

Os cursos de graduação de Turismo c Hotclaria no Brasil tiveram origen na década de 1970, especificamente na cidade de São Paulo. Até 1976 não ultrapassavam dez cm todo o país, mas, apesar da crise dos anos vilcuti. proliferaram e hoje somam mais de quarenta cursos. Além desses, nos anos nov'cnta aparecem os cursos de pós-graduação (striclo sensu e lato sen.ul) ․ll Turismo.

Por ocasião de realização de eventos, desenvolvimento de pesquisas c outras atividades cientificas na área, tiveram-se inúmeras dificuldades em ter acesso a um "mailing" atualizado das escolas brasilciras que ministram esses cursos. Mesmo consul tando professorese entidades daárea, as listagens is quais se tiveram acesso cram desatualizadas c incompletas. Considerou-se, assim. quis um esforço de levantamento deveria ser feito a fim de se identificar o universo desses cursos no Brasil.

Mediante a colaboração de professores c especialistas ligados a entıdarkes educacionais e de classe em Turismo e Hotelaria ${ }^{3}$, levantaram-se inicialmento

1 Bacharel em Tiurismo pela Faculdade Ideal de Letras e Ciencias Humanas. Doutora em ( Timil da Comunicação (Turismo) pela Escola de Comunicações e Artes da USP. Pesyuliadít conveniada e professora colaboradora na ECAMISP. Professora Titular e Coordenthitr. Wh Curso de Turismo da Universidade Paulista.

End. para corresp.: R. Min. Américo Marco Antonio, 204-0 05442-040 - São Paul. ¿ ‘f.

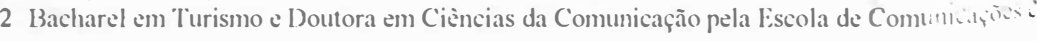
Artes da USP. Professora dos cursos de graduação e pós-graduação da E.CA/USP'.

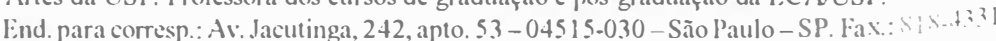

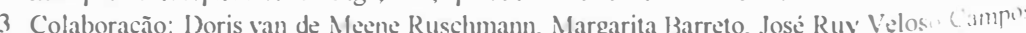
Ciguelzaco do AVI ENBE:TUR - Encontro de Bacharéis e Estudantes de Turismo. ercal de trinta cursos de graduação. Após isso, solicitaram-se informações sobre outros cursos através de carta, fax e contato telefonnico, chegando a um total de 41 cursos de graduação, 1 de pós-graduação em nívelde mestrado (stricto sensu)

e 12 de pós-graduação em nível deespecializaçãoe aperfeiçoamento (lalo sensu).

Pclos dados coletados, observou-se que existe apenas 1 curso de pósraduação siriclo sensu específico na área: o Mestrado em Turismo e Lazer da Escola de Comunicações e Artes da Universidade de São Paulo.

Com relação aos cursos em nível de especialização constatou-se a existência de 12 em funcionamento neste ano, pois como não são cursos regulares poderão ou não ser oferecidos nos próximos anos. A maioria desses cursos $(58.3 \%)$ concentra-se na cidade de São Paulo, sendo que os restantes $(41,6 \%)$ cstão distribuídos por Natal $(R N)$, Salvador (BA), Barbacena (MG), Rio de Janeiro (RJ) e Balneário de Camboriú (SC); enfocam desde o Turismo e Lazer sob o ponto de vista macro, até temas mais específicos, como gerência de empresas turísticas, administração hoteleira e de eventos, gestão de negócio em alimentação, planejamento c marketing, e turismo ambiental

Os cursos de graduação encontram-se distribuídos em 15 Estados do Brasil (Tabela 1). A maioria desses cursos (78\%) é de Turismo, seguindo-se os de Hotclaria (19,5\%). Há um único curso de Turismo e Hotelaria, que começou

a funcionar cm 1990, em função das necessidades de formação e capacitação de recursos humanos para o Turismo e Hotelaria na região. A distribuição desses zursos por região (Figura 1) mostra maior concentração na Região Sudeste $(51,2 \%)$, seguida da Região Nordeste (22\%) e Sul (19,5\%); as menores concentrações situam-se na RegiãoNorte $(4,8 \%)$ eRegião Centro-Oeste $(2,5 \%)$.

A scguir, apresenta-se a relação dos cursos levantados, por tipo/Estado. No caso dos cursos de graduação, arrolaram-se, também, os cursos que sc iniciaram no segundo semestre destc ano ou que terão início em 1995. 
Tabela 1 - Cursos de Graduação em Turismo e Hotelaria no Brasil x Estado - 1994

\begin{tabular}{|c|c|c|c|}
\hline \multirow[b]{2}{*}{ Estado } & \multicolumn{3}{|c|}{ Cursos de Graduaçāo } \\
\hline & $\begin{array}{c}\text { Turismo } \\
n^{\circ}\end{array}$ & $\begin{array}{c}\begin{array}{c}\text { Ilotelaria } \\
n^{\circ}\end{array} \\
\end{array}$ & $\begin{array}{c}\text { Tur/Hot. } \\
\mathbf{n}^{\circ}\end{array}$ \\
\hline Amazonas & 1 & 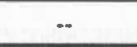 & -- \\
\hline Pará & 1 & - & -- \\
\hline Maranhão & 1 & I & -- \\
\hline Ceará & 1 & - & - \\
\hline Rio Grande do Norte & 2 & -- & -- \\
\hline Peniambuco & 2 & -. & -- \\
\hline Bahia & 1 & I & -- \\
\hline Distrito Federal & I & -- & -- \\
\hline Minas Gerais & I & -- & -- \\
\hline Espirito Santo & I & - & -- \\
\hline Rio de Janeiro & 5 & 1 & $-\cdots$ \\
\hline São Paulo & 10 & 3 & -- \\
\hline Paraná & 2 & -- & -- \\
\hline Santa Catarina & -- & -- & 1 \\
\hline Rio Grande do Sul & 3 & 2 & $\because$ \\
\hline TOTALPorcentagem & $32(78,0 \%)$ & $8(19,5 \%)$ & $1\left(2,5^{\bullet} 0\right)$ \\
\hline
\end{tabular}

Nota: Dados levantados em agosto de 1994.

Figura 1 - Cursos de Graduação em Turismo e Hotelaria x Região (Brasil, 1994).

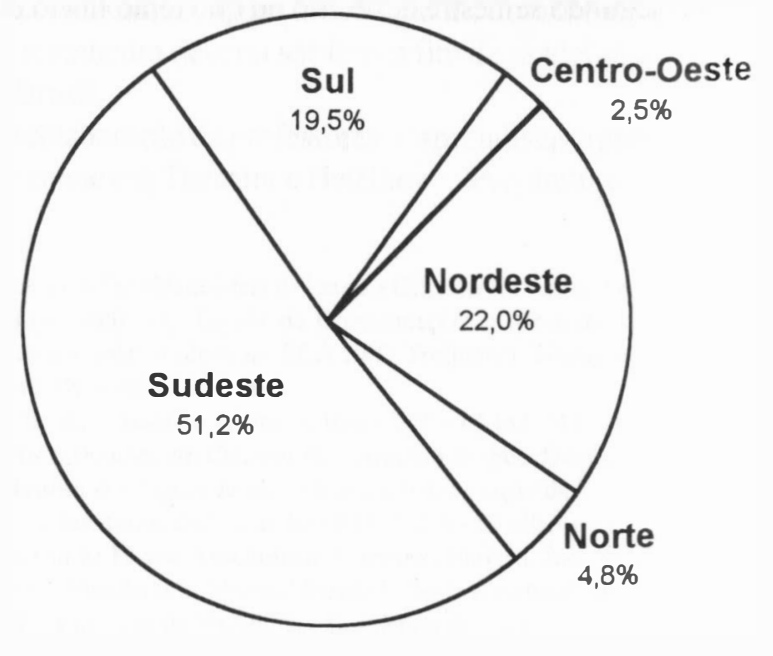

\section{CURSOS DE GRADUAÇÃO EM TURISMO E HOTELARIA}

\subsection{Turismo}

- Estaclo do Amazonas

S()(II:I)ADE EDUCACIONAL DE MANAUS

CLRSO DE TURISMO

DIRETT(OR: Prof. Walderi Areosa Ferreira

C(OORIJINADOR: Profa. Maria Augusta Ponce de Leão

Rua lirei l.ourenço, 33 -69010-470 - Manaus - AM

Tel.: (092) 633-2144-Fax.: (092) 232-453

Nota: primeiro vestibular em 13/08/1994

\section{- Estado da Bahic}

ASSOCIACCÃO CULTURAL E EDUCACIONAL DA BAHIA FAC'LI.DADE DE TURISMO DA BAHIA

CO()RIDENADOR: Prof. Edilene Barbosa - A/C Profa. Carmélia Anna Amaral

Rua da Mangueira, 32 - Nazaré - 40040-400 Salvador - BA

Tel.: (1)71 ) 243-8828/1973/4999 - Fax.: (071) 321-9143

\section{- Estaclo do Cearo}

(NINTERSIDADE DE FORTALEZA - UNIFOR - FUNDAÇÃO EDSON QUEIROZ CLRS() DE TURISMO

C()()RIDENADOR: Prof. Antonio Marçal Pinto de Castro

Av: W:ashington Soares, 1321 - 60811-341 - Fortaleza - CE

Tel.: (085) 273-2833 ramal 153 - Fax.: (085) 273-1667

- Distrito Federal

(NIIÃO) PIONEIRA DE INTEGRAÇÃO SOCIAL - UPIS FAC. DI: CIÊNCIAS EXATAS, ADMIN. E SOCIAIS DE BRASILIA CL'RS() DE TURISMO

COORIDENADOR: Prof EDSON FERREIRA NETO

SEPLEOS - 712/912 - MÓDULO A - 70390-125 - Brasilia - DF

Tel. : (061) 3461944

- Estado do Espirito Santo

ASS()(IAÇÃO DE ENSINO SUPERIOR DE GUARAPARI FAC'L'IIDADE DE TURISMO DE GUARAPARI

COORIDENADOR: Profa. Marleidi Mocelin de Oliveira 
Rodovia Jones dos Santos Neves, 1000 - Bairro Lagoa Funda 29200-000 - Guarapari - ES

Tel.:(027) 261-2821 - Fax.: (027) 361-0195

\section{- Estado do Maranhão}

UNIVERSIDADE FEDERAL DO MARANHÃO - CENTRO DE ESTUDOS BÁSICOS CURSO DE TURISMO

CAMPUS UNIVERSITÁRIO DO BACANGA

COORDENADORA: Profa. Maria do Socorro Araújo

Bloco Centro de Estudos Básicos - Av. dos Portugueses s/

65080-420 - São Luis - MA

Tel.: (098) 232-3466 ramal 162 - Fax.: (098) 222-3186

\section{- Estado de Minas Gerais}

NSTITUTO MINEIRO DE CIÉNCIAS ADMINISTRATIVAS E TECNOLÓGICAS FACULDADE DE TURISMO DE BELO HORIZONTE

CHEFE DO DEPTo. DE ESTUUDOS TURISTICOS: Prof. Celso M Machado

COORDENADOR: Profa. Mary Elizabeth Pereira Pinto

Rua Goitacazes, 1762 - Bairro Preto/Centro

30190-052 - Belo Horizonte - MG

Tel.: (03I) 295-1922 - Fax.: (031) 295-3916

\section{- Estado do Para}

UNIVERSIDADE FEDERAL DO PARA

CAMPUS UNIVERSITÁRIO DO GUAMA -CENTRO SOCIOECONÔMICO CURSO DE TURISMO

COORDENADOR DO COLEGIADO: Prof. José Lúcio B.do Nascimento

Rua Augusto Correa s/n - 66050-040 - Belém - PA

Tel.: (091) 249-2088 ramal:385/219 - Fax. (091) 229-9677

\section{- Estado do Parana}

FACULDADE DE CIÉNCIAS SOCIAIS APLICADAS DE FOZ DO IGUAÇ CURSO DE TURISMO

COORDENADOR: Prof. Marco Antonio Sena de Souz

Rua "D" s/n (Vila Sảo José) - Antigo Colégio Anglo-Americano

85867-530 - Foz do Iguaçu - PR

Tel.: (0455) 22-2211/522-3222 - Fax.: (0455) 22-2221
NIVERSIDADE FEIERAL DO PARANA - UFPR

CURSO DE TURISMO

CIIEFE DO DEPTO. DE COMUNICAÇÃO SOCIAL: Prof. Miguel Bah

C()ORDENADOR: Prof. Eduardo Manoel Marques Pereira

Trav. Alfredo Bufrem, 140 - 20 - 80020-240 - Curitiba - PR

Eav: (041) 264-22791

\section{- Estado de Pernambuco}

UNIVERSIDADE CATÓLICA DE PERNAMBUCO - UNICAP DEPARTAMENTO DE COMUNICAÇÃO SOCIAL

CURSO DE TURISMO

C()(ORDENADOR: Profa. Georgina Cavalcanti

Rua do Principe, 526 - Campus Universitário- Boa Vista

50050-900 - Recife - PE

Tel.: (081) 216-4145 - Fax.: (081) 216-4204/231-1842

UNIVERSIDADE FEDERAL DE PERNAMBUCO CURSO DE TURISMO

PR()-REITOR ACADĖMICO(substituto): Prof. Josenildo do Santos

Av. Prof. Moraes Rego, 1235 - 50732-970 - Recife - PE

Cidade Universitária - Campus USPE

Tel.: (081) 271-8101/271-8100 - Fax.: (081) 271-8099

Nota: primeiro vestibular em dezembro/1994

- Estado do Rio Grande do Norte

FACULDADE PARA EXECUTIVOS - FACEX

CURSO DE TURISMO

CO()RDENADOR: Prof. Luiz Carlos Cardoso D'Avila

Rua Orlando Silva, 2897 - 59012-500 - Natal - RN

Tel.: (084) 217-5851 - Fax.: (084) 217-5851

FACULDADE UNIFICADA PARA O ENSINO DAS CIÊNCIAS - UNIPEC CURSO DE TURISMO

C(OORDENADORA: Profa. Jurema Marcia Dantas da Silva

R. Floriano Peixoto, 295 - 59012-500 - Natal - RN

Tel.: (084) 221-5090 - Fax.: (084) 221-6255

- Estado do Rio Grande do Sul

FUNDAÇÃO UNIVERSIDADE DE CAXIAS DO SUL - UCS

ESCOLA SUPERIOR DE HOTELARIA E TURISMO

CURSO DE TURISMO

DIRETOR: GILBERTO BONATTO

CO()RDENADOR: Profa. Maria Helena Marques

Rua Rodolfo Schllipier, 222 - Caixa Postal, 81 -95680-000 - Canela - RS 
PONTIFICIA UNIVERSIDADE CATÓLICA DO RIO GRANDE DO SUL ACULDADE DOS MEIOS DE COMUNICAÇÃO SOCIAL

CURSO SUPERIOR DE TURISMO

COORDENADOR: Profa. Norma Moesch

Av. Ipiranga, 6681 - Azenha - Jardim Botânico - 90160-092 - Porto Alegre - RS Tel.(051) 339-1511 ramal 3269 - Fax ·(051)336-9400/339-1564

Tel.(054) 282-1714/282-1156 - Fax.:(054) 282-1156

UNIVERSIDADE LUTERANA DO BRASIL - ULBRA ESCOLA SUPERIOR DE HOTELARIA DE CANELA CURSO DE TURISMO DO CAMPUS DE TORRES

COORDENADOR: Profa. Luciane de Carvalho Pereira

Estrada Municipal s/n - 95560-000 - Torres - RS

Tel.: (051) 664-1421 - Fax.: (051) 664-2800

\section{- Estado do Rio de Janeiro}

FACULDADE DA CIDADE

CURSO DE TURISMO

CHEFE DO DEPTO. DE TURISMO: Profa. Maria Gema Moreir

Av. Epitácio Pessoa, 1664 - 3o andar - Lagoa - 22471-000 - Rio de Janeiro - RJ Tel.: (021) 287-1145 - Fax.: (021) 287-8941

FACULDADES INTEGRADAS HELIO ALONSO - FACHA

CURSO DE TURISMO

COORDENADOR: Prof. Fernando Maia Tavares

Rua Muniz Barreto, 51 - 22251-090 - Rio de Janeiro - RJ

Tel.: (021)551-5695/5448/5645/5096 - Fax.: (021) 551-5096

FACULDADES INTEGRADAS PLINIO LEITE

FACULDADE NITEROIENSE DE EDUCAÇÃO, LETRAS E TURISMO

CURSO DE TURISMO

CHEFE DO DEPARTAMENTO DE TURISMO: Profa. Diana Vaidman

R. Visc. do Rio Branco, 123 a 137 - 24020-000 - Niterói - RJ

Tel.: (021) 622-1441 ramal 331 - Fax:: (021) 717-8922

UNIVERSIDADE ESTÁCIO DE SÁ

CURSO DE TURISMO

DIRETOR DO DEPTO. DE TURISMO E HOTELARIA: Prof. Ruy Otávio

Bernardes de Andrade

COORDENADOR: Profa. Célia Maria Cortez

Rua do Bispo, 83 - 20261-060 - Rio de Janeiro - RJ

Tel.: (021) 503-7000 - Fax.: (021) 293-4539
UNIVERSIDADE VEIGA DE ALMEIDA - UVA

CURSO DE TURISMO

CHEFE DO DEPTO. DO CURSO DE TURISMO: Profa. Tania G. Omena Rua lbiturana, 108 - BI. B - 40. andar - Maracanã -

20271-020 - Rio de Janeiro - RJ

Tel.: (021) 264-6172 - Fax.: (021) 248-2165

- Estado de São Paulo

ASSOCIAÇÃO EDUCACIONAL DE ENSINO SUPERIOR FACULDADE RIOPRETENSE DE COMUNICAÇÃO SOCIAL

CURSO DE TURISMO

COORDENADOR: Profa. Maria Lúcia Gabrie

Rua Eduardo Nielson, 960 - Jardim Aeroporto - 15030-070 -

São José do Rio Preto - São Paulo - SP

Tel.: (0172) 33-7910/34-3787 - Fax.: (0172) 34-3787

NOTA: primeiro vestibular em 1995

ASSOCIAÇÃO EDUCACIONAL DO LITORAL SANTISTA

CURSO DE TURISMO

COORDENADOR: Prof José Manuel Dias

Rua Rangel Pestana, 99 - 11013-551 - Santos - SP

Tel.: (0132) 32-2556 - Fax.: (0132) 32-7078

FACULDADE ANHEMBI MORUMB

CURSO DE TURISMO

COORDENADOR: Profa. Gracira Cabrera

Rua Casa do Ator, 90 - 04546-000 - São Paulo - SP

Tel.:(0II) $821-9020$ - Fax.:(0II) $821-9547$

FACULDADE CAPITAL DE ADMINISTRAÇÃO E ESTATISTICA

CURSO DE TURISMO

COORDENADOR: Prof Marlene Mathis

Rua Ibipetuba, 42 - Moóca - 03127.180 - São Paulo - SP

Tel.: (01 I) 273-5011/273-5214 - Fax.: (011) 273-2650

FACULDADE IBERO-AMERICANA DE LETRAS E CIÊNCIAS HUMANAS CURSO DE TURISMO

COORDENADOR: Prof. Haroldo Leitão Camargo

Av. Brig. Luis Antonio, 871 - 01317-00I - São Paulo - S

Tel.: (011) 37-0071 - Fax.: (01I) 35.3385

ORGANIZAÇÃO SANTAMARENSE DE EDUCAÇÃO E CULTURA - OSEC CURSO DE TURISMO

A/C Profa. Rita de Cássia Giraldi Santin

Rua Prof. Enéas de Siqueira Neto, 340 - Jardim Imbuias

04829-300 - São Paulo - SP

Tel. (01I) 520-9611 ramal 330 - Fax. (011) \$20-9160 
PONTIFICIA UNIVERSIDADE CATÓLICA DE CAMIPINAS

INSTITUTO DE ARTES E COMUNICAÇÕES

CURSO DE TURISMO

VICE-DIRETORA DO IAC E COORDENADORA DO DEPTO. DE TURISMO Profa. Maria Ângela A. Bissoli

COORDENADOR: Prof. Luiz Gonzaga Godoi Trigo

Rodovia Dom Pedro I, Ḱm 136 - Ed. Papa VI - Campus I

Caixa Postal 317 - 13100-904 Campinas - SP

Tel.: (0192) 52-0899 ramal 176 - Fax.: (0192) 52-8477

UNIVERSIDADE DE FORMAÇÃO EDUCACIONAL E CULTURAL - UNIFEC CURSO DE TURISMO

COORDENADOR: Prof: Ricardo Pedreira Désio

Rua Amazonas, 2000 - 09540-203 - São Caetano do Sul - SP

Tel.: (011) 441-3233 ramal 225 - Fax.: (011) 441-4220

UNIVERSIDADE PAULISTA - UNIP

CURSO DE TURISMO

COORDENADOR: Profa. Marilia Gomes dos Reis Ansarah

Av. Paulista, 900 - 01310-100 - São Paulo - SP

Tel.: (011) 253-7700 ramal 123 ou 180 - Fax.: (011) 251-0171

UNIVERSIDADE DE SÃO PAULO - USP

ESCOLA DE COMUNICAÇÕES E ARTES

CURSO DE TURISMO

COORDENADOR: Profa. Sarah S. Bacal

Av. Prof. Lúcio Martins Rodrigues, 443, Bloco B - 05508-900 - São Paulo - SP

Tel.: (01) 818-4082/4122 - Fax.: (0I1) 818-4331/814-4764

\subsection{Hotelaria}

- Estado da Bahia

CENTRO FEDERAL DE EDUCAÇÃO TECNOLOGICA DA BAHIIA - CEFET-BA CAMPUS UNIVERSITÁRIO S/N - PITANGUINHA

CURSO TECNOLOGO EM ADMINISTRAÇÃO HOTELEIRA

COORDENADOR: Profa. Eliana Maria Oliveira da Hora

AVC PEDRO PAULINO SOUTO

3700-000 - Simões Filho - Bahi

Tel.: (071) 396-8400 - Fax.: (071) 396-7326
- Estado do Maranhão

UNIVERSIDADE FEDERAL DO MARANHÃO

CENTRO DE ESTUDOS BÁSICOS

CURSO DE TECNOLOGIA EM IIOTELARIA

COORDENADOR: Prof. José Gomes de Aguiar

Av. dos Portugueses s/n, Campus Bacanga - Bloco Centro de Estudos Básicos -

65080-420 - São Luís - MA

Tel.: (098) 232-3666 ramal 193 - Fax.: (098) 222-8684

- Estado do Rio Grande do Sul

CENTRO DE ESTUDOS TURISTICOS E HOTELEIROS - CETI CURSO DE IIOTELARIA

DIRETOR/COORDENADOR: Prof. Geraldo Castelli -

Rua Dona Carlinda, 370 - Centro

Caixa Postal 181 - 95680-000 - Canela - RS

al: (054) 282-1460 - Fax. (054) 282-1460

95680-000 - CANELA - RIO GRANDE DO SUL

FUNDAÇÃO UNIVERSIDADE DE CAXIAS DO SUL - UCS

ESCOLA SUPERIOR DE IIOTELARIA E TURISMO

CURSO DE HOTELARIA

DIRETOR: Prof: Gilberto Bonatto

CAIXA POSTAL, 81 - Centro - Rua Rodolfo Schllipier, 222 -95680-000 - Canela - RS Tel.: (054) 282-1714/282-1156

\section{- Estado do Rio de Janeiro}

UNIVERSIDADE ESTÁCIO DE SÁ

CURSO DE HOTELARIA

DIRETOR DO DEPTO. DE TURISMO E HOTELARIA:

Prof. Ruy Otávio Bemardes de Andrade

COORDENADOR: Profa. Maria Tereza Maldonado

Rua do Bispo, 83 - 29261-060 - Rio de Janeiro - RJ

Tel.: (021) 503-7000 - Fax.:(021) 293-4339

\section{- Estado de São Paulo}

FACULDADE DE EDUCAÇÃO, CIÊNCIAS E LETRAS HEBRAICO-RENASCENCCA CURSO DE HOTELARIA

COORDENADOR: Profa. Aparecida Rodrigues Lacerda

Rua São Vicente de Paula, 650 - Iligienópolis - $01229-010$ - São Paulo - SP

Tel.: (011) 824-0788 - Fax.: (011) 825-1837 
FACULDADE IBER()-AMERICANA DE LETRAS E CIENCIAS HUMANAS CURSO DE ADMINISTRAÇAOIOIIOTELLEIRA

COORDENAD()R: Prof. Haroldo Leitão Camargo

Av. Brig. Luis Antonio, 87I - 01317-00 I - São Paulo - SI

Tel.: (011) 37-0071 - Fax: (011) 35-3385

Nota: vestibular em janeiro de 1995

SERVIÇO NACIONAL DE APRENIDLZAGEM COMERCIAL - SENAC/CEATEL. CURSO DE TECNOLOGIA EM IIOTELARIA

DIRETOR: Prof. José Ruy Veloso Campos-

Av. Francisco Matarazzo, 249 - 05001-150 - São Paulo - SP

Tel.: (011) 263-5804/2404/2511 - Fax.: (011) 864-4597

\subsection{Turismo c Hotelaria}

- Estado de Santa Catarina

INIVERSIDADE DO VAI.E D() ITAJA

FACULDADE DE TURISMO E IICTELARIA - FATUHVI

DIRETOR: Profa. Marlene Buratto

Rua 5a. Avenida, Bairro dos Municipios - Caixa Postal 20

88330-000 - Balneário Camboriu - SC

Tel.: (0473) 67-1613 - l:ax.: (0473) 67-1613

\section{CURSO DE POS-GRADUACCÃO STRIC'TO SENSU EM TURISMO}

- Estaclo de Sĩ̃o Paulo

(INIVERSIDADE DE SÃ() PAULO

ESCOLA DE COMUNICAÇÕES E ARTES

DEPARTAMENT() IJE RELAÇÕES PÚBLICAS, PROPAGANDA E TURISMO MESTRADO EM TURISM() E LAZER

COORDENADOR: Profa. Sara Strachmann Bacal

Av. Prof. Lúcio Martins Rodrigues, 443 - Bloco B - 05508-900 - São Paulo - SP

Tel.: (011) 818-4122/818-4147 - Fax.: (011) 818.4331

\section{CURSOS DE PÓS-GRAdUAÇÃO LATO SENSU EM LAZER,}

\section{TURISMO E HOTELARIA}

- Estado da Bahia

ASSOCIAÇÃO CULTURAL, EDUCACIONAL DA BAHIA

CENTRO DE ESTUIDOS DE PÓS-GRADUAÇÃO OLGA METTIG

FACULDADE DE TURISMO DA BAHHIA
CURSO DE ESPECIALIZAÇÃO EM TURISMO

COODENADOR: Profa. Eny C. Vasconcelos e Maria de L. Marques

Rua da Mangueira, 32 - Nazaré - 40040-400 - Salvador - BA

Tel.: (071) 243-8826 - Fax:: (071) 321-9143

- Estado de Mfinas Gerais

SER VICO NACIONAL DE APRENDIZAGEM COMERCIAL - SENAC/CEATEL (en convènio com a UNIVERSIDADE FEDERAL DE JUIZ DE FORA)

CURSO DE PÓS-GRADUAÇÃO EM ADMINISTRAÇÃO HOTELEIRA

COORDENADOR: Prof. Celso Antonio Dalla Costa

Av. Cruz das Almas, sin - 36.200-000 - Barbacena - $M O$

- Estado do Rio Cirande do Norte

FACULDADE UNIFICADA PARA O ENSINO DAS CIÊNCIAS - UNIPEC

CURSO DE GERENCIA DE EMPRESAS TURISTICAS

COORDENADOR: Profa. Dra. Françoise Dominique Valery

R. Floriano Peixoto, 295 - $59012-500$ - Natal - RN

Tel.: (084) 211.5090 - Fax: (084) 221-625

- Estado do Rio de Janeiro

FACULDADES INTEGRADAS PLINIO LEITE

CURSO DE PLANEJAMENTO NA ÁREA DE TURISMO

CHEFE DO DEPARTAMENTO DE TURISMO Profa. Diana Vaidman

R. Visc. do Rio Branco, 123 a 137 - 24020-000 - Niterói - RJ

Tel.: (021) 622-1441 ramal 351 - Fax.: (021) $717-8922$

- Estado de Santa Catarina

UNIVERSIDADE DO VALE DO ITAJAI

FACULDADE DE TURISMO E HOTELARIA - FATUHVI

CURSO DE TURISMO E HOTELARIA

DIRETORA: Profa. Marlene Buratto

COORDENADOR: Profa. Dra. Doris van de Meene Ruschmann

Rua 5a. Avenida, Bairro dos Municipios - Caixa Postal 201

88330-000 - Balneário Camboriu - SC

Tel.: (0473) 67-1613 - Fax.: (0473) 67-1613

- Estado de Sã̃o Paulo

FACULDADE DE EDUCACÃO, CIÊNCIAS E LETRAS HEBRAICO-RENASCENÇA CURSO DE ADMINISTRACÃOHOTELEIRA

COORDENADOR: Prof. Jacob Puternan

Rua São Vicente de Paula, 650 - 01229-010 - São Paulo - SP

Tel.: (011) 824-0788 - Fax: (011) 825-1837 
FUNDAÇÃO ESCOLA DE SOCIOLOGIA E POLÍTICA CURSO DE ADMINISTRAÇÃO DE CENTROS DE LAZER

COORDENADOR: Prof. Luiz Octávio de Lima Camargo

Rua Gen. Jardim, 522 - 01223-010 - São Paulo - SP

Tel.: (011) 255-2001/256-6393 - Fax.: (011) 222-9680

SER VIÇO NACIONAL, DE APRENDIZAGEM COMERCIAL - SENAC/CEATEL DIRETOR: Prof. José Ruy Veloso Campos

Av. Francisco Matarazzo, 249 - 05001-150 - São Paulo - SP

Tel.: (011) 263-2404/263-2511 - Fax.: (011) 864-4597

- CURSo de administraÇÃo de EVENTOOS

COORDENADOR: Acadêmico: Profa. Cristiane Paz Fernandez Técnico: Prof. Margarida Lameirinhas

- CURSO DE ADMINISTRAÇÃo hOTELEIRA COORDENADOR: Acadêmico: Profa. Cristiane Paz Fernandez Técnico: Prof. Wladir Vieira Duar

- CURSo de gestão de nEGócio em alimentaÇÃo de COLETIVIDAdE COORDENADOR: Acadêmico: Profa. Cristiane Paz Fernandez Técnico: Profa. Maria Suely Ferreira Valcov

- CURSO DE PLANEJAMENTO E MARKETING TURÍstico COORDENADOR: Acadêmico: Profa. Cristiane Paz Fernandez Técnico: Prof. Dra. Doris van de M. Ruschmann

- curso de turismo ambiental COORDENADOR: Acadêmico: Profa. Cristiane Paz Fernandez Técnico: Prof. Luiz Wilson Pina 